Benchmarks

\title{
A modified feeding RNAi method for simultaneous knock-down of more than one gene in Caenorhabditis elegans
}

Kyoengwoo Min ${ }^{1, * * *}$, Junsu Kangl, ${ }^{1,}$, and Junho Lee ${ }^{1,2}$

${ }^{1}$ Research Center for Functional Cellulomics, Institute of Molecular Biology

and Genetics, School of Biological Sciences, Seoul National University, Seoul, Korea, and ${ }^{2}$ WCU program, Department of Biophysics and Chemical Biology, Seoul National University, Seoul, Korea

BioTechniques 48:229-232 (March 2010) doi 10.2144/000113365

Keywords: C. elegans; feeding RNA interference; double knock-down

Supplementary material for this article is available at www.BioTechniques.com/article/113071.

${ }^{*}$ K.M. and J.K. contributed equally to this article.

**K.M.'s present address is Department of Cellular Biology, University of Georgia Athens, GA, 30602, USA.

RNA interference (RNAi) is a commonly used technique for reverse genetic approaches in Caenorhabditis elegans. Feeding RNAi is the most convenient and inexpensive method for performing genome-wide RNAi screens. However, it has been reported that knock-down of two genes (double RNAi) by feeding RNAi using a mixture of bacteria that each contained one dsRNA species produced poor results. To overcome this problem of inefficiency, we designed and tested a double feeding RNAi method using a single RNAi construct containing two gene fragments. From experiments with three different sets of genes, we found that the new double RNAi method consistently produced significantly enhanced double knock-down phenotypes. The double feeding RNAi approach described here provides a method to consistently examine phenotypes caused by depletion of more than one gene in C. elegans.

Reverse genetics using RNA interference (RNAi) has become a major tool in biological research after its first discovery in the nematode Caenorhabditis elegans (1). Although there are a few tissues in C. elegans that are refractory to RNAi, systemic RNAi can be accomplished by simply delivering double-stranded RNA (dsRNA) into any part of the body of $C$. elegans, since it has the ability to transport dsRNA across cell boundaries (2). Several methods have been used for delivering RNAi, including microinjecting dsRNA into worm body tissue (injection RNAi), soaking worms in dsRNA-containing solution (soaking RNAi), and feeding bacteria expressing dsRNA (feeding RNAi) (1-3). Among them, feeding RNAi has become the favored method because it is the most convenient and inexpensive (Figure 1A, left panel). Production of genome-wide collections of feeding RNAi bacteria made genome-wide RNAi-based screening possible in $C$. elegans $(4,5)$. A drawback of feeding RNAi is that, in contrast to the injection RNAi method, the knock-down of more than one gene by feeding RNAi may produce poor results $(4,6)$. Worms fed a mixture of two kinds of bacteria-each expressing dsRNA for one gene-often show greatly reduced phenotypes for each gene (Figure 1A, middle panel) (7). Limitations in one or more components of the RNAi machinery are commonly believed to cause problems when multiple dsRNA species are introduced, but it is likely that other factors limit the efficiency of the double feeding
RNAi method as well. We hypothesized that decreased quantities of each dsRNA species delivered to the animals would result in a dilution effect that reduces the manifestation of the corresponding phenotype (8). When compared with the amount of dsRNA delivered to worms in single RNAi, only $50 \%$ of dsRNAs for each gene would be delivered in double feeding RNAi using the mixture of bacteria, since the total number of bacteria ingested by a worm would be the same. A simple solution to this limitation would be to feed worms bacteria expressing dsRNA for two genes within single cells by using a single vector containing DNA fragments from both genes, instead of feeding two kinds of bacteria (Figure 1A, right panel). With this method, the same amount of dsRNA for each gene can be delivered into worms.

To examine the efficiency of this new method, we first chose two target genes for double feeding RNAi, $g f p$ and $b l i-1$, whose phenotypes are obvious and easy to score. We assessed the efficiency of feeding double RNAi by feeding $C$. elegans bacterial cells carryingdsRNAs for both genes, compared with feeding worms two separate bacteria that each carried dsRNA for a single gene. We fed dsRNA-expressing bacteria to a nematode strain containing an integrated daf-16::gfp transgene, TJ356 (9). The bli-1 gene encodes a collagen involved in adult cuticle formation $(10,11)$. Knock-down of bli-1 using feeding RNAi with bacteria from the feeding RNAi library (5) resulted in a strong blistered phenotype with the efficiency of $90 \%$ (Figure 1B, Table S1). However, when bacteria that expressed dsRNA of either $b l i-1$ or $g f p$ were mixed and fed to worms, the proportion of Bli worms was reduced to $30 \%$. In contrast, the knock-down efficiency of $g f p$ was only slightly lower than that of single RNAi, suggesting that not all genes are sensitive to the dilution effect. To test the efficiency of feeding multiple genes in a single vector, we generated an RNAi construct containing both $b l i-1$ and $g f p$ in a single vector. The worms fed with bacteria expressing dsRNA for $b l i-1$ and $g f p$ showed strong double RNAi phenotypes including a Bli phenotype and no GFP signal as the result of successful knock-down of both genes (Figure 1B, Table S1). Since only bli-1 RNAi showed the dilution effect, we examined whether either bli-l or $g f p$ showed the dilution effect when they were mixed with control bacteria containing the empty vector L4440. When the bli-1 RNAi bacteria were diluted with the control bacteria, we observed a dosedependent weaker phenotype (Figure 1C, 
A

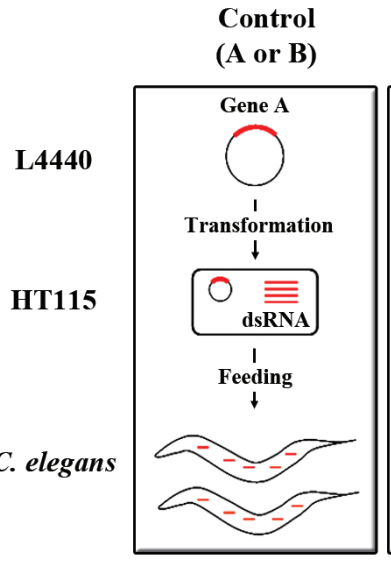

B

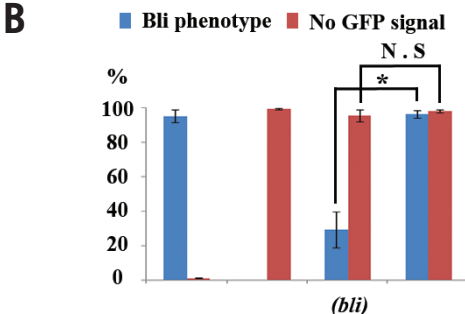

D

RNAI bli-1 gfp
RNA $i \quad b l i-1$
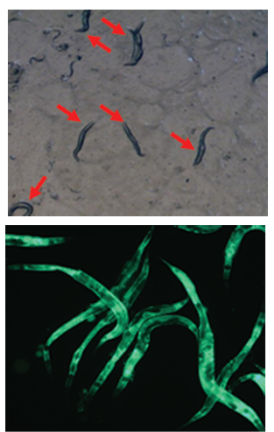

Multiple vectors

(A)+(B)

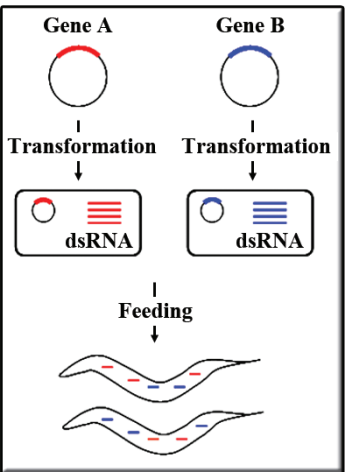

C

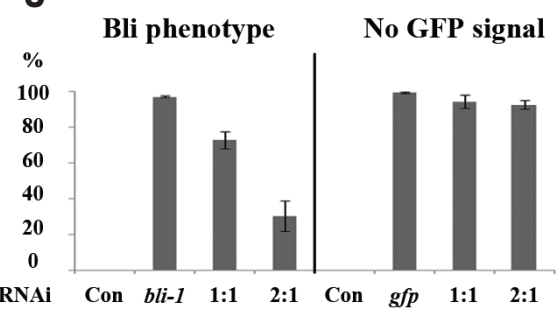
(gfp)

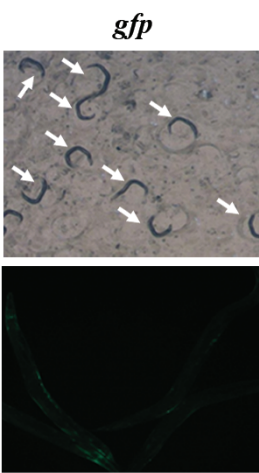

$(b l i)+(g f p)$

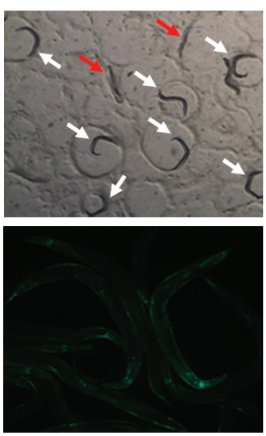

Single vector (A+B)

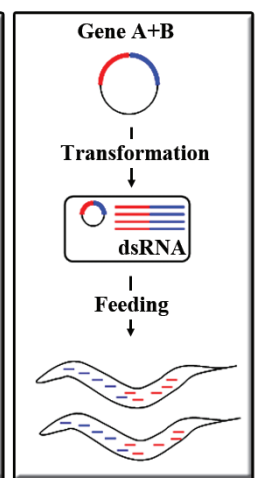

Figure 1. The modified feeding RNAi method in C. elegans. (A) A schematic diagram of the modified feeding RNAi method. Left panel, the conventional single feeding RNAi; middle panel, double feeding RNAi with a mixture of two bacteria; right panel, the newly demonstrated double feeding RNAi method using a bacterial strain expressing dsRNA for two genes. I (B) Average numbers of worms that showed Bli phenotype (blue) and no GFP fluorescence signal (red). ${ }^{*}, P<0.01$. N.S., not significant. (C) Dilution effect of bli-1 and gfp feeding RNAi. (D) The Bli phenotype (top) and GFP phenotype (bottom) resulting from feeding RNAi for the genes indicated. The red arrows indicate worms showing the Bli phenotype and the white arrows indicate worms showing the non-Bli phenotype. Sets of parentheses indicate individual bacterial strains expressing the dsRNA of the indicated gene(s).

Table S2). However, $g f p$ RNAi did not show any dilution effect, consistent with the experiment described above. These data suggest that RNAi of some genes, but not all, showed the dilution effect and that using a single vector expressing two dsRNA species is a way to effectively avoid dilution and achieve RNAi effects.

To examine whether the new method is also applicable to endogenous genes, we tested the RNAi effects of the $d p y-5$ and/or mes-3 genes. The dpy-5 gene encodes cuticle collagen. When it is silenced by RNAi, the resulting worms display a Dumpy phenotype (11). mes-3 is a maternal-effect gene that, when silenced by RNAi, produces sterile progeny (12). Single RNAi feeding against $d p y-5$ or mes-3 was effective for producing each phenotype. However, when worms were fed with a mixture of two kinds of bacteria (each expressing dsRNA for either $d p y-5$ or mes-3), worms did not show the Dpy phenotype. In contrast, the knock-down different from that seen with single RNA $\mathrm{i}$ (Figure 2A, Table S3). When worms were fed bacteria expressing $d p y-5$ and mes-3 efficiency of mes-3 was not significantly
dsRNA contained in a single vector, the proportion of Dpy worms increased significantly (Figure 2A, Table S3).

We also tested the genes oma-1 and oma-2, which encode functionally redundant zinc fingers. The loss of both genes causes the Oocyte Maturation defective (Oma) phenotype, which is easily visible under a microscope (13). The depletion of either oma-1 or oma-2 did not cause the Oma phenotype (Figure 2B, Table S3). However, depletion of both genes-by feeding either a mixture of two kinds of bacteria, each expressing one dsRNA, or by feeding a single bacteria expressing both genes in one vector-caused the Oma phenotype. We then fed worms the dpy-5 dsRNA-expressing bacteria together with bacteria expressing dsRNA for the two oma genes and found that the Dpy phenotype was decreased considerably, while the Oma phenotype was not affected. In contrast, almost all worms fed bacteria expressing dsRNA for all three genes in single cells showed the Dpy phenotype (Figure 2B, Table S3). The orders and orientations of the inserts did not affect the RNAi efficiency. With these data, we suggest that the new method is also suitable for endogenous genes, especially in cases where double feeding RNAi with two separate bacteria is ineffective for either one of the genes.

In this study, we were able to accomplish the efficient knock-down of two genes simultaneously by a simple modification to the existing feeding RNAi strategy. Our results clearly showed that feeding RNAi with a DNA construct containing two or three gene fragments in one plasmid is efficient and reliable in knocking down the functions of the genes. The method described here may not be necessary for genes that show phenotypes by feeding two separate kinds of bacteria, but this method provides a good means of RNA interference for genes exhibiting reduced RNAi efficiency due to dsRNA dilution from bacteria expressing another dsRNA. While performing RNAi in a $C$. elegans strain with enhanced RNAi sensitivity (e.g., $r r f-3$ or eri-1;lin-15B) would be one way to improve double RNAi efficiency (6), knock-down of the two genes simultaneously in a single vector would be another good alternative to examine phenotypes caused by the disruption of two genes. Our method can be more useful for those who are concerned that a genetic background with enhanced RNAi (Eri) sensitivity might alter the phenotypes they wish to study. 


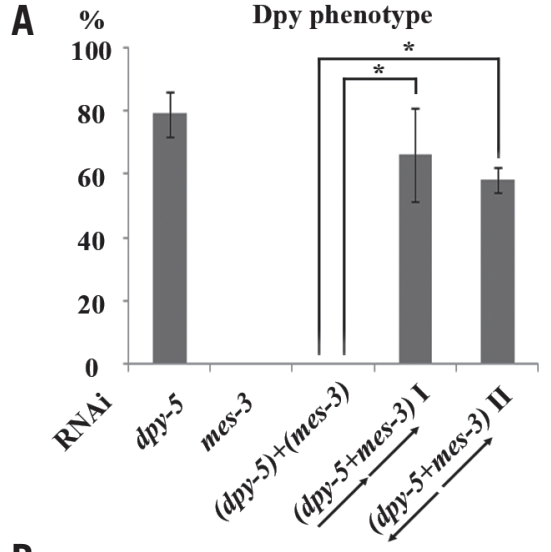

B

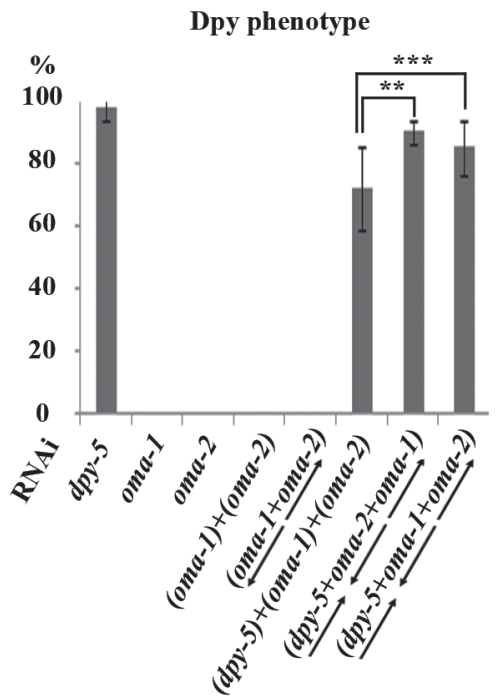

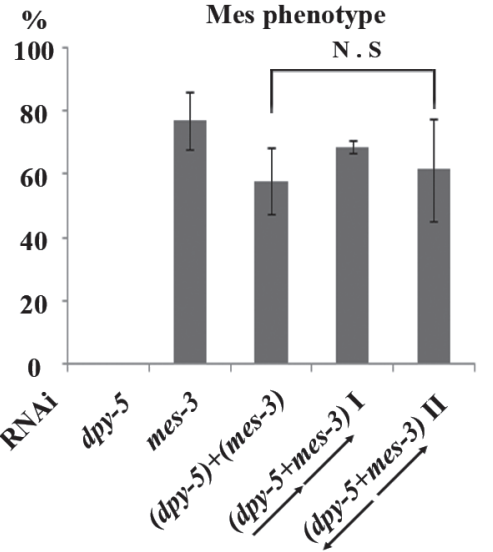

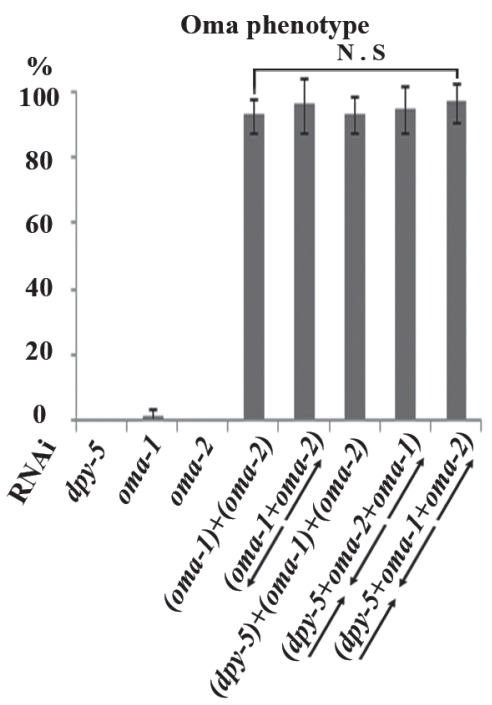

Figure 2. Application of the modified feeding RNAi method to endogenous genes. (A) Quantification of worms that showed the Dpy (left panel) and the Mes (right panel) phenotypes. Parentheses indicate individual bacterial strain expressing the dsRNA of indicated gene(s). Arrows indicate the 5'-to-3' direction of inserts. (B) Quantification of worms that showed the Dpy (left panel) and the Oma (right panel) phenotypes. Parentheses indicate individual bacterial strains expressing the dsRNA of the indicated gene(s). The order of the genes written corresponds to their insertion within the vector. ${ }^{*}, P<0.001 ; * *, P<0.002$; and ${ }^{* * *}, P<0.05$. N.S., not significant.

\section{Acknowledgments}

We thank Andrew Fire (Stanford University, Palo Alto, CA, USA) for the nematode expression vectors. This work was supported by funding from the Research Center for Functional Cellulomics at Seoul National University, the Korea Science and Engineering Foundation (KOSEF), a Molecular and Cellular BioDiscovery Research Program grant from the Ministry of Science and Technology (grant no. 20090083336), and a grant from the National R\&D Program for Cancer Control, Ministry for Health, Welfare and Family Affairs (grant no. 0920230).K.M. was supported by a postdoctoral grant from the Korean Research Foundation (KRF; grant no.
2005-037-C00027), the Brain Korea 21 (BK21) program (2007), and the Seoul R\&BD Program (2007).

\section{Competing interests}

The authors declare no competing interests.

\section{References}

\footnotetext{
1.Fire, A., S. Xu, M.K. Montgomery, S.A. Kostas, S.E. Driver, and C.C. Mello. 1998. Potent and specific genetic interference by double-stranded RNA in Caenorhabditis elegans. Nature 391:806-811.

2. Tabara, H., A. Grishok, and C.C. Mello. 1998. RNAi in C. elegans: soaking in the genome sequence. Science 282:430-431.
}

3. Timmons, L. and A. Fire. 1998. Specific interference by ingested dsRNA. Nature 395:854.

4. Fraser, A.G., R.S. Kamath, P. Zipperlen, M. Martinez-Campos, M. Sohrmann, and J. Ahringer. 2000. Functional genomic analysis of C. elegans chromosome I by systematic RNA interference. Nature 408:325-330.

5. Kamath, R.S. and J. Ahringer. 2003. Genome-wide RNAi screening in Caenorhabditis elegans. Methods 30:313-321.

6. Ahringer, J., ed. 2006. Reverse genetics. In WormBook: The online review of $C$. elegans biology. doi/10.1895/wormbook.1.47.1, http://www.wormbook.org.

7. Kamath, R.S., M. Martinez-Campos, P. Zipperlen, A.G. Fraser, and J. Ahringer. 2001. Effectiveness of specific RNA-mediated interference through ingested doublestranded RNA in Caenorhabditis elegans. Genome Biol. 2:RESEARCH0002.

8. Parrish, S., J. Fleenor, S. Xu, C. Mello, and A. Fire. 2000. Functional anatomy of a dsRNA trigger: differential requirement for the two trigger strands in RNA interference. Mol. Cell 6:1077-1087.

9. Henderson, S.T. and T.E. Johnson. 2001. daf-16 integrates developmental and environmental inputs to mediate aging in the nematode Caenorhabditis elegans. Curr. Biol. 11:1975-1980.

10. Brenner, S. 1974. The genetics of Caenorhabditis elegans. Genetics 77:71-94.

11. Page, A.P. and I.L. Johnstone. 2007. The cuticle. In WormBook: The online review of $C$. elegans biology.doi/10.1895/wormbook.1.138.1, http://www.wormbook.org.

12.Paulsen, J.E., E.E. Capowski, and S. Strome. 1995. Phenotypic and molecular analysis of mes-3, a maternal-effect gene required for proliferation and viability of the germ line in C. elegans. Genetics 141:13831398.

13. Detwiler, M.R., M. Reuben, X. Li, E. Rogers, and R. Lin. 2001. Two zinc finger proteins, OMA-1 and OMA-2, are redundantly required for oocyte maturation in $C$. elegans. Dev. Cell 1:187-199.

Received 28 October 2008; accepted 6 January 2010.

Address correspondence to Junho Lee, School of Biological Sciences, Seoul National University, 56-1 Gwanak-gu, Seoul, Korea 151-742. e-mail: elegans@snu.ac.kr 